\title{
QUIMIOPROFILAXIA DA MALÁRIA COM MEFLOQUINA NA AMAZÔNIA BRASILEIRA
}

\author{
João Barberino Santos, Aluízio Prata e Eduardo Wanssa
}

\begin{abstract}
Em estudo randômico duplo-cego, 122 voluntários morando em área endêmica de malária na Regiäo Amazônica (Estado de Rondônia) foram divididos em quatro grupos para estudo da supressão malárica. 0 grupo I recebeu $500 \mathrm{mg}$ de mefloquina a cada quatro semanas; o grupo II $250 \mathrm{mg}$ de mefloquina a cada duas semanas; o grupo Ill um comprimido de Fansidar (500mg de sulfadoxina $+25 \mathrm{mg}$ de pirimetamina) por semana e o grupo IV, recebeu apenas placebo. Um ataque agudo de malária ocorreu em um individuo do grupo I, em dois individuos do grupo II e em seis individuos dos grupos III e IV. A proteçao verificada nos grupos em uso de mefloquina foi significantemente superior comparada ao grupo placebo. A mefloquina, em ambas as dosagens usadas, mostrou-se efetiva na supressão malárica em uma área onde o Plasmodium falciparum plurirresistente é altamente prevalente.
\end{abstract}

Palavras-chaves: Malária. Mefloquina. Quimioprofilaxia.

As tentativas de controle em massa do paludismo humano na Amazônia têm-se assentado fundamentalmente no controle ao transmissor alado, através das campanhas de borrifação com inseticidas organoclorados e organofosforados. Esse contínuo esforço, desenvolvido pela Fundação Nacional de Saúde (FNS), tem encontrado inúmeros obstáculos à erradicação da malária na região, resultando apenas impactos parciais e passageiros no controle da endemia.

A quimioprofilaxia tem sido tentada em experiências isoladas, temporárias e limitadas a determinados agrupamentos humanos, usando diversos tipos de drogas. $O$ ensaio mais extenso e o de maior duração feito no Brasil, foi executado na década de 50 por Pinotti e Soares ${ }^{24}$ com sal cloroquinado. O método, no entanto, foi abandonado por dificuldades técnicas no seu emprego e pelo receio de que a sua tomada irregular provocasse o surgimento e a dispersão de raças de Plasmodium falciparum cloroquino-resistentes.

Com efeito, a difusão de raças de Plasmodium falciparum plurirresistentes na Amazônia tem sido um fato notoriamente já comprovado ${ }^{1727}$ que vem aumentar a limitação da quimioprofilaxia de

Núcleo de Medicina Tropical e Nutrição da Universidade de Brasília, Brasília, DF.

Endereço para correspondência: Dr. João Barberino Santos. Centro de Pesquisa e Tratamento de Malária do Vale do Guaporé. Av. Limoeiro s/n. 78971-000 Costa Marques, RO. Recebido para publicação em 15/05/91. uma área tão vasta e de difícil acesso.

$O$ aparecimento de novos antimaláricos de uso simplificado, com eficiente ação esquizonticida contra raças plurirresistentes e, praticamente isentode efeitos colaterais, reabre a possibilidade de se poder contar com um quimioprofilático eficiente na proteção de indivíduos expostos à transmissão malárica em uma área endêmica.

A mefloquina, DL-erytro-2,8-bis (trifluoromethyl)-alfa-(2-piperidyl)-4quinolinemethanol hydrocloridrato, foi sintetizada em 1971 pelo Instituto Walter-Reed, com relevante sucesso no tratamento da malária plurirresistente em várias partes do mundo 9112528 .

$O$ presente trabalho representa o primeiro teste quimioprofilático com a mefloquina no Estado de Rondônia, situada na Amazônia Ocidental Brasileira, considerada região malarígena hiperendêmica e, também, caracterizada como área de Plasmodium falciparum cloroquino-resistente, cujos resultados preliminares já foram apresentados anteriormente ${ }^{26}$.

\section{MATERIAL E MÉTODOS}

O estudo foi desenvolvimento entre agosto de 1982 e janeiro de 1983 com voluntários soldados e

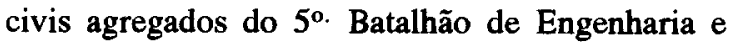
Construção, acampados na localidade conhecida como Corintianas, às margens da BR 364 que liga Porto Velho, ROà Cuiabá, MT, na época, um trecho ainda em fase de desmatamento e de terraplenagem. 
Santos JB, Prata A, Wanssa E. Quimioprofilaxia da malária com mefloquina na Amazônia Brasileira. Revista da Sociedade Brasileira de Medicina Tropical 26:157-162, jul-set, 1993.

De início, foram selecionados 120 voluntários, 30 para cada grupo de estudo. Seis indivíduos foram logo excluídos após a triagem inicial e não foram computados. Oito indivíduos deixaram a área de estudo quando já estavam sendo acompanhados (1 após a $10^{\mathrm{a}}$ semana e 7 após a $11^{\mathrm{a}}$ semana) suas observações foram computadas e eles foram substituídos por 8 novos voluntários. Assim, com 6 desistências e 8 substituições, o número total de pessoas participantes foi igual a 122 .

Os participantes eram do sexo masculino, com idade variando entre 15 e 54 anos. No entanto, por exceção, 3 mulheres de 15, 25 e 26 anos, fora do período de gestação, ficaram incluídas no grupo I do estudo. A maioria dos participantes já tinha meses ou anos de residência na área, com vários episódios pregressos de malária e todos podem ser considerados como semi-imunes. Foram excluídos da triagem as crianças com menos de 12 anos, gestantes, idosos acima de 55 anos, pessoas com doenças debilitantes, pessoas que haviam tomado antimaláricos nas últimas 4 semanas antes de iniciar o estudo e pessoas alérgicas às sulfonamidas.

$O$ estudo foi randômico, duplo-cego. Semanalmente, no dia determinado para cada indivíduo, eram ingeridos 4 comprimidos de igual aspecto, contidos em envelopes lacrados e com um código determinado para cada indivíduo que só foi aberto após terminado o ensaio. Os comprimidos de placebo tinham aspecto idêntico ao das drogas ativas e completavam um número de 4 quando o número de comprimidos da droga experimentada era menos de quatro nos envelopes correspondentes. Cada comprimido de mefloquina continha $250 \mathrm{mg}$ da base e cada comprimido de Fansidar, 500mg de sulfadoxina $+25 \mathrm{mg}$ de pirimetamina.

Os 122 participantes foram divididos em 4 grupos: o grupo I ( 31 indivíduos) recebeu $500 \mathrm{mg} \mathrm{de}$ mefloquina a cada 4 semanas; o grupo II (32 indivíduos) recebeu $250 \mathrm{mg}$ de mefloquina a cada 2 semanas; o grupo III ( 29 indivíduos) recebeu um cromprimido de Fansidar por semana; o grupo IV (30 indivíduos) recebeu apenas placebo.

À triagem inicial, em cada visita semanal e ao fim do estudo, o indivíduo era questionado sobre doenças, principalmente sobre sintomas e sinais compatíveis com malária. Uma amostra de sangue era tomada para determinação do hematócrito, contagem de leucócitos e para esfregaço corado pelo método de Giemsa para pesquisa de hematozoários em 400 campos microscópicos em cada lâmina examinada.

o tempo médio de observação e acompanhamento das pessoas em cada grupo (Tabela 1) foi o seguinte:

$\begin{array}{ll}\text { Grupo I } & 17,5+/-6,2 \text { semanas } \\ \text { Grupo II } & 17,1+/-6,8 \text { semanas } \\ \text { Grupo III } & 17,6+/-7,3 \text { semanas } \\ \text { Grupo IV } & 15,5+/-7,7 \text { semanas }\end{array}$

As causas de exclusão durante o estudo foram: mudança da área para outro local, perda de comprimidos, episódio de malária aguda e reações adversas.

Tabela 1 - Duraçao do tratamento supressivo da malária falciparum em quatro grupos de indivfduos usando mefloquina em duas dosagens, Fansidar e placedo.

\begin{tabular}{rrrrr}
\hline $\begin{array}{c}\text { Duração do } \\
\text { tratamento } \\
\text { (semanas) }\end{array}$ & $\begin{array}{c}\text { Grupo } \\
\text { I }\end{array}$ & $\begin{array}{c}\text { Grupo } \\
\text { II }\end{array}$ & $\begin{array}{c}\text { Grupo } \\
\text { III }\end{array}$ & $\begin{array}{c}\text { Grupo } \\
\text { IV }\end{array}$ \\
\hline$<5$ & - & 1 & 1 & 3 \\
$5-9$ & 5 & 4 & 4 & 4 \\
$10-14$ & 4 & 8 & 6 & 6 \\
$15-19$ & 8 & 3 & 1 & 5 \\
$20-24$ & 14 & 16 & 17 & 12 \\
\hline Total & 31 & 32 & 29 & 30 \\
\hline
\end{tabular}

\section{RESULTADOS}

Os casos de malária aguda ocorridos em cada grupo se distribuíram da seguinte forma:
Grupo I 1 por $P$. falciparum
Grupo II 2 por malária mista
Grupo III 3 por $P$. falciparum
1 por $P$. vivax
2 por malária mista
$\begin{array}{ll}\text { Grupo IV } & 2 \text { por } P \text {. vivax } \\ & 4 \text { por malária mista }\end{array}$

As diferenças entre as incidências dos ataques agudos de malária nos grupos de indivíduos tratados com as drogas antimaláricas não foram estatisticamente diferentes do grupo tratado com placebo, pelo teste de Fischer $p>0,05$. No entanto, as diferenças entre os grupos tratados com mefloquina e o grupo placebo foi significante pelo 
teste de Fischer $\mathrm{p}<0,05$.

$\mathrm{O}$ momento de ocorrência do ataque palúdico durante o período de execução da profilaxia, pode ser visto na Tabela 2.

Tabela 2 - Momento do aparecimento do ataque agudo de malária durante o estudo da profilaxia com a mefloquina em duas diferentes doses, com Fansidar e com placebo.

\begin{tabular}{|c|c|c|c|}
\hline Grupo & $\mathrm{N}^{0}$ de casos & $\begin{array}{c}\text { Tipo } \\
\text { de } \\
\text { Malária }\end{array}$ & $\begin{array}{l}\text { Tempo de } \\
\text { aparecimento } \\
\text { após o início } \\
\text { da profilaxia } \\
\text { (semanas) }\end{array}$ \\
\hline I & 1 & P. faciparum & 8 \\
\hline \multirow[t]{2}{*}{ II } & 1 & mista & 5 \\
\hline & 1 & mista & 5 \\
\hline \multirow[t]{6}{*}{ III } & 1 & P. falciparum & 8 \\
\hline & 1 & P. falciparum & 13 \\
\hline & 1 & P. falciparum & 18 \\
\hline & 1 & P. vivax & 2 \\
\hline & 1 & mista & 12 \\
\hline & 1 & mista & 6 \\
\hline \multirow[t]{6}{*}{ IV } & 1 & P. vivax & 15 \\
\hline & 1 & P. vivax & 5 \\
\hline & 1 & mista & 2 \\
\hline & 1 & mista & 6 \\
\hline & 1 & mista & 5 \\
\hline & 1 & mista & 7 \\
\hline
\end{tabular}

Efeitos colaterais - reações adversas ocorreram em 1 paciente de cada grupo (Tabela 3 ). As principais reações foram de nervosismo, irritabilidade, malestar e dor epigástrica de intensidade discreta a moderada. Em relaçãoà mefloquina, é mais provável relacioná-las com o uso da droga quando esta foi usada em maior dosagem (500mg de 4/4 semanas).

Não houve nenhuma alteração do hematócrito em nenhum dos quatro grupos.

Observou-se discreta diminuição da contagem dos leucócitos antes e durante o tratamento em muitos indivíduos nos quatro grupos. No entanto, em nenhum dos participantes houve uma alteração importante do número de leucócitos, de forma que a leucopenia não foi constatada em nenhuma das ocasiões em que foi checada.

Tratamento de doenças concomitantes - dois pacientes, ambos do grupo II, sofreram doenças concomitantes: em um, houve queimaduras na terceira semana de estudo e foi tratado com tetraciclina por 16 dias; em outro, foram detectadas amebíase e ascaridíase, tratado na décima semana com tinidazol e levamizole. Esses pacientes não foram excluídos do estudo.

\section{DISCUSSÃO}

Tanto o uso de 500mg de mefloquina a cada 4 semanas, quanto o de $250 \mathrm{mg}$ a cada 2 semanas, mostrou-se efetivo na supressão da malária em uma área onde a malária falciparum multirresistente é

Tabela 3 - Distribuição das reações colaterais nos indivíduos em tratamento supressivo da malária com mefloquina (em duas dosagens) e com Fansidar.

\begin{tabular}{|c|c|c|c|c|c|c|}
\hline Grupo & $\mathbf{N}^{\mathbf{O}}$ de indiv. & $\begin{array}{l}\text { Tipo de } \\
\text { reação }\end{array}$ & Severidade & $\begin{array}{l}\text { Tempo de } \\
\text { tratam. } \\
\text { (semanas) }\end{array}$ & $\begin{array}{l}\text { Duração } \\
\text { (dias) }\end{array}$ & $\begin{array}{c}\text { Relação com a } \\
\text { droga }\end{array}$ \\
\hline \multirow[t]{2}{*}{ I } & 1 & nervosismo & discreta & 2 & 2 & possível \\
\hline & & mal-estar & discreta & 2 & 2 & possível \\
\hline \multirow[t]{2}{*}{ II } & 1 & fadiga & discreta & 9 & 2 & remota \\
\hline & & mal-estar & discreta & 9 & 2 & remota \\
\hline \multirow[t]{3}{*}{ III } & 1 & nervosimo & moderada & 1 & 3 & possível \\
\hline & & irritabilidade & moderada & 2 & 3 & possível \\
\hline & & gastralgia & moderada & 1 & 3 & possível \\
\hline
\end{tabular}


Santos JB, Prata A, Wanssa E. Quimioprofilaxia da malária com mefloquina na Amazônia Brasileira. Revista da Sociedade Brasileira de Medicina Tropical 26:157-162, jul-set, 1993.

altamente prevalente.

$\mathrm{Na}$ literatura, tem sido recomendada a dose de $250 \mathrm{mg}$ de mefloquina por semana para profilaxia ${ }^{4}$ 31. Porém, nos trabalhos de Felix e cols ${ }^{11}$, a meia vida da mefloquina foi avaliada em mais de 20 dias e, em 21,6 dias, em média, por De Souza e cols ${ }^{8} \mathrm{em}$ pacientes brasileiros semi-imunes. Com base em nossos resultados, concluímos que o espaço de tempo entre as administrações da droga poderá ser mais dilatado, sem prejuízo do efeito supressivo.

As triagens clínicas com a mefloquina conduzidas no Brasil na década de 80 , mostraram uma alta eficácia terapêutica desta droga, entre $98 \%$ e $100 \%^{2}$. No entanto, relatos esporádicos de malária mefloquino-resistente têm ocorrido principalmente na África e no Sudeste Asiático ${ }^{2} 34512151621$ inclusive resistência cruzada entre mefloquina e halofantrine ${ }^{10}$. Diante disto, é posta em dúvida a validade da profilaxia com a mefloquina naquelas regiões ${ }^{21}$.

No intuito de retardar a emergência de raças do Plasmodium falciparum resistentes à mefloquina, tem sido preconizada a profilaxia com a associação mefloquina-sulfadoxina-pirimetamina (MSP) ${ }^{32}$. Entretanto, além dos temidos efeitos colaterais dos compostos sulfônicos ${ }^{42}$, já ocorre na Amazônia Brasileira a resistência do Plasmodium falciparum à combinação sulfadoxina-pirimetamina ${ }^{1227}$, o que pode ter sido a razão das falhas encontradas neste estudo no grupo que usou Fansidar, comparativamente iguais às do grupo placebo. Desta forma, existe o risco potencial de aparecimento de cepas resistentes à combinação MSP e, o seu emprego como profilático de massa nas áreas endêmicas não deve ser preconizado.

A mefloquina foi bem tolerada e as reações colaterais foram mínimas e infreqüentes, em concordância com outros estudos realizados com esta droga 46792226 . A baixa freqüência de efeitos colaterais com a mefloquina sozinha e a sua eficácia como profilático, comparada com o Fansidar, sugerem que ela possa ser usada nas áreas plurirresistentes pelos viajantes que visitam estas áreas por tempo limitado ${ }^{413}$. Há suspeitas de que a quimioprofilaxia prolongada nos programas de controle, possa causar depressão das gamaglobulinas séricas, interferindo no desenvolvimento dos anticorpos antiplasmódios nas populações protegidas ${ }^{14}$. Por outro lado existe o temor de difusão da resistência à droga: experimentalmente, Peters e cols ${ }^{20}$ observaram uma tendência da resistência à mefloquina na malária de roedores, sob pressão da droga. Na Amazônia Venezuelana foram encontradas cepas de $P$. falciparum resistentes in vitro à mefloquina ${ }^{18}$. Além disso, existe a possibilidade de resistência cruzada entre a mefloquina e outras drogas como, por exemplo, ao halofantrine ${ }^{10}$. Recentemente, tem aparecido na literatura relatos sobre sérias reações adversas neurológicas e psiquiátricas causadas pelo uso profilático da mefloquina, com maior risco em pessoas com história de doença epiléptica e maníacodepressiva ${ }^{23}{ }^{30}$. A mefloquina não deve ser indicada como profilático para pessoas morando em áreas endêmicas.

Na Amazônia Brasileira, onde a mefloquina não foi comercializada e o seu uso tem se restringido à terapêutica, sob o controle da FNS, concordando com o pensamento de Sturchler ${ }^{29}$, a mefloquina pode ser considerada ainda uma excelente opção profilática indicada apenas ao uso restrito de viajantes para as áreas de transmissão por tempo limitado, em casos especiais a serem decididos por especialistas. Com base nos resultados aqui obtidos, as doses profiláticas recomendadas seriam de $500 \mathrm{mg}$ de mefloquina a cada 4 semanas ou, de $250 \mathrm{mg}$ a cada 2 semanas.

\section{SUMMARY}

In a randomised double blind study 122 volunteers living in an endemic malarious area in Amazonian Rondonia state were divided into 4 groups to study malaria supression. The first group received 500 $\mathrm{mg}$ of mefloquine every month, group II $250 \mathrm{mg}$ every two weeks, group III a tablet of Fansidar (500mg sulphadoxine $+25 m g$ pyrimethamine) $a$ week and group IV placebo. Acute attacks of malaria occured in one individual in group I, 2 subject in group II, and 6 individuals in groups III e IV. Protection with mefloquine was significant compared with the placebo group. Both treatment regimens with mefloquine were effective supressants in an area of high prevalence of drug multiresistant Plasmodium falciparum transmission.

$$
\text { Key-words: Malaria. Mefloquine. }
$$

Chemoprophylaxis. 


\section{AGRADECIMENTOS}

Os autores agradecem aos Laboratórios Roche pelo fornecimento da mefloquina e auxílio para realização deste trabalho. Também, expressam seu agradecimento ao Comando do $5^{\circ}$. Batalhão de Engenharia e Construção, Porto Velho, RO por colocar-nos à disposição o seu laboratório e instalações de campo.

\section{REFERÊNCIAS BIBLIOGRÁFICAS}

1. Alecrim MGC. Estudo da resistência do Plasmodium falciparum às drogas anti-maláricas in vivo e in vitro na Amazônia. Tese de Mestrado em Medicina Tropical. Universidade de Brasília, Brasília, 1981.

2. Bjorkman A, Phillips-Howard PA. The epidemiology of drug-resistant malaria. Transactions of the Royal Society of Tropical Medicine and Hygiene 84:177. $180,1990$.

3. Brasseur P, Konamano J, Moyon RS, Druilhe P. Emmergence of mefloquine-resistant malaria in Africa without drug pressure. The Lancet 336:59, 1990.

4. Brecknridge A. Risks and benefits of prophylactic antimalarial drugs. British Medical Journal 299:1057$1058,1989$.

5. Chippaux JP, Massougbodji A, Akogbeto M, Josse R, Zohoun T, Sadeler BC. Evolution de la chimiosensibilité de Plasmodium falciparum a la chloroquine et a la méfloquine au Bénin entre 1980 et 1989. Bulletin de la Societé de Pathologie Exotique 83:320-329, 1990.

6. Clyde DF, Mc Carthy VC, Miller RM, Hornick RB. Supressive activity of mefloquine in sporozoiteinduced human malaria. Antimicrobial Agents and Chemotherapy 9:384-386, 1976.

7. De Souza JM. Mefloquine clinical trials Therapeutical experience with mefloquine alone and in combination (MSP) in brazilian male subject with falciparum malaria. Memórias do Instituto Oswaldo Cruz 81(supl II):259-268, 1986.

8. De Souza JM, Heizmann P, Schwartz DE. Singledose kinetics of mefloquine in Brazilian male subjects. Bulletin of the World Health Organization 65:353356, 1987.

9. Doberstin EB, Phintuyothin P, Noeypatimanondh $S$, Teerakiartjamjorn C. Single-dose therapy of falciparum malaria with mefloquine or pyrimethamine-sulfadoxine. Bulletin of the World Health Organization 57:275-278, 1979.

10. Felix R, Gay F, Lyogoubi A, Bustos MDG, Diquet B, Davis M, Gentilini M. Résistance croiseé a la mefloquine et a l'halofantrine lors d'un paludisme a Plasmodium falciparum contracté en Sierra Leone. Bulletin de la Societé de Pathologie Exotique 83:4345, 1990.

11. Felix H, Gentilin M. La mefloquine-Rapport preliminaire. In: Resums de le Deuxiéme Congrés de la Societé Mediterranéenne de Chimiotherapie, Nice, France, 1980.

12. Gay F, Binet MH, Bustos MDG, Rrouveix B, Danis $M$, Roy $C$, Gentilini M. Mefloquine failure in child contracting falciparum malaria in west Africa. The Lancet 355:120-121, 1990.

13. Gilles HM. The treatment and prophylaxis of malaria. Annals of Tropical Medicine and Parasitology 81:607-617, 1987.

14. Greenwood BM. The impact of malaria chemoprophylaxis on the immune status of Africans. Bulletin of the World Health Organization 62(suppl):69-75, 1984.

15. Karwacki JJ, Webster HK, Limsonwong N, Shanks GD. Two cases of mefloquine resistant malaria in Thailand. Transactions of the Royal Society of Tropical Medicine and Hygiene 83:152-153, 1989.

16. Kiliami VAEB, Mkufya AR, Kilama WL. Low resistance of Plasmodium falciparum to mefloquine in Tanga region Tanzania. Transactions of the Royal Society of Tropical Medicine and Hygiene 83:162$164,1989$.

17. Kremsner PG, Zotter GM, Feldmeier H, Bienzle V, Jansea-Rosseck R, Graninger W, Rocha $M$, Wernsdorfer WH. Differences in drug response of Plasmodium falciparum within an area of the Amazon Region. Transactions of the Royal Society of Tropical Medicine and Hygiene 83:158-161, 1989.

18. Maynaidé M, Peceño C, Noriega P-L, Yarzabal L. Susceptibility of Plasmodium falciparum strains to chloroquine and mefloquine in the Amazonas Federal Territory of Venezuela. Transactions of the Royal Society of Tropical Medicine and Hygiene 83:586$588,1989$.

19. Nosten F, Terkuile F, Chongsuphajaisiddhi T, Luxemburger C, Webster HK, Edstein M, Phaipun L, Kyan LT, White NJ. Mefloquine-resistant falciparum malaria on the Thai-Burnese border. The Lancet 337:1140-1143, 1991.

20. Peters W, Portus J, Robinson BL. The chemotherapy of rodent malaria. XXVIII, The development of resistance to mefloquine (WR 142,190). Annals of Tropical Medicine and Parasitology 71:419-426, 1977.

21. Petersen E, Hogh B, Bygbjerg IC, Black FT. Malaria chemopropylaxis: Why mefloquine? The Lancet 336:811, 1990.

22. Peto TEA, Gilks CF. Strategies for the prevention 
Santos JB, Prata A, Wanssa E. Quimioprofilaxia da malária com mefloquina na Amazônia Brasileira. Revista da Sociedade Brasileira de Medicina Tropical 26:157-162, jul-set, 1993.

of malaria in travellers: comparison ofdrug regimens by means of risk-benefit analysis. The Lancet I:12561261, 1986.

23. Phillips-Howard PA, Bjorkman AB. Ascertainment of risk of serious adverse reactions associated with chemoprophylatic antimalarial drugs. Bulletin of the World Health Organization 68:493-504, 1990.

24. Pinotti $\mathbf{M}$, Soares R. A erradicação da malária com sal cloroquinado. Revista Brasileira de Malariologia e Doenças Tropicais 8:253-265, 1956.

25. Ponnapaham JT. Mefloquine in the treatment of malaria a preliminary study. Abstract of the 10th Congress of Tropical Medicine and Malaria. Manila, Philippines p.9-15, 1980.

26. Prata A, Santos JB, Ferreira I, LeimerR. Supressive treatment of malaria in the Amazon Region. In: Abstracts of the XI International Congress of Tropical Medicine and Malaria. Calgary (Canadá) p.122, 1984.

27. Reyes S, Osanai CH, Passos ADC. Resistência in vivo do Plasmodium falciparum às 4-amino- quinoleinas e à associação sulfado xina-pirimetamina . II - Estudo de Manaus, Amazonas 1983-1984. Revista Brasileira de Malariologia e Doenças Tropicais 38:37-44, 1986.

28. Santos JB, Prata A. Tratamento de 75 pacientes portadores de malária pelo $P$. falciparum em Porto Velho (RO), com mefloquina. Revista da Sociedade Brasileira de Medicina Tropical 21:181-185, 1988.

29. Sturchler D. Malaria prophylaxis in travellers: the current position. Experientia 40:1357-1362, 1984.

30. Sturchler D, Handschin J, Kaiser D, Kerr L, Mittelholzer ML, Reber $\mathbf{R}$, Fernex $M$. Neuropsychiatric side effects of mefloquine. The New England Journal of Medicine 333:1752-1753, 1990.

31. Wernsdorfer WH. Current approaches to malaria chemotherapy and prophylaxis. Parasitology Today 2:220-253, 1986.

32. World Health Organization. Advances in malaria chemotherapy. Technical Repports Series $n^{0} \cdot 711$, Geneva, 1984. 